Pacific

Journal of

Mathematics

NORMS ON THE COHOMOLOGY OF A 3-MANIFOLD AND SW THEORY

Stefano Vidussi 


\title{
NORMS ON THE COHOMOLOGY OF A 3-MANIFOLD AND SW THEORY
}

\author{
SteFAnO VidUssi
}

\begin{abstract}
The aim of this paper is to discuss some applications of the relation between Seiberg-Witten theory and two natural norms defined on the first cohomology group of a closed 3manifold $N$ - the Alexander and the Thurston norm. We will start by giving a "new" proof, applying SW theory, of McMullen's inequality between these two norms, and then use these norms to study two problems related to symplectic 4-manifolds of the form $S^{\mathbf{1}} \times N$. First we will prove that - as long as $N$ is irreducible - the unit balls of the Thurston and Alexander norms are related in a way that is similar to the case of fibered 3-manifolds, supporting the conjecture that $N$ has to be fibered over $S^{\mathbf{1}}$. Second, we will provide the first example of a 2-cohomology class on a symplectic manifold (of the form $S^{\mathbf{1}} \times N$ ) that lies in the positive cone and satisfies Taubes" "more constraints", but cannot be represented by a symplectic form, disproving a conjecture of $\mathrm{Li}$ and $\mathrm{Liu}(\mathrm{Li}-\mathrm{Liu}$, 2001, Section 4.1).
\end{abstract}

\section{Introduction.}

It has been proven, in [McM], that two natural (semi) norms defined on the first cohomology group of a 3-manifold, namely the Alexander norm $\|\cdot\|_{A}$, defined from the Alexander polynomial of the manifold, and the Thurston norm $\|\cdot\|_{T}$, defined in terms of the minimal genus of the representatives of the Poincaré dual two dimensional homology class, satisfy a relation expressed in the following:

Theorem 1.1 (McMullen). Let $N$ be a compact, connected, oriented 3manifold (eventually with boundary a union of tori); then the Alexander and Thurston norm satisfy

$$
\|\cdot\|_{A} \leq\|\cdot\|_{T}+ \begin{cases}\left(1+b_{3}(N)\right) \operatorname{div}(\cdot) & \text { if } b_{1}(N)=1 \\ 0 & \text { if } b_{1}(N)>1,\end{cases}
$$

where $\operatorname{div}(\cdot)$ denotes the divisibility of an element in $H^{1}(N, \mathbb{Z})$. 
This inequality, applied to the particular case where the three manifold is the exterior of a knot $K$, reduces to the well-known fact that the degree of the Alexander polynomial of the knot (i.e., the difference between highest and lowest power) is bounded from above by twice the genus of the knot, i.e., the lowest value of the genus of a Seifert surface of the knot.

These two norms turn out to be strictly related to the 3-dimensional Seiberg-Witten theory.

The proof of Theorem 1.1 given in $[\mathbf{M c M}]$ is purely topological, but it is suggested the existence of a proof based on SW theory. (It appears that the first one to observe this has been D. Kotschick; P. Kronheimer previously proved the inequality in the case of $N$ obtained as 0 -surgery of a knot, in [K2]; the first detailed proof of the general case appeared in a preprint of the author $([\mathbf{V}])$, on which this paper is partly based.)

Our first aim will be to write the two norms in terms of SW basic and monopole classes for $N$. This allows, as mentioned, an alternative proof of Theorem 1.1, that we will work out for the case, for us more interesting, of a closed manifold with $b_{1}(N)>1$.

Then we will use these results to study symplectic 4-manifolds of the form $S^{1} \times N$, for an irreducible $N$. We will prove the following:

Theorem 1.2. Let $N$ be an irreducible 3 -manifold with $b_{1}(N)>1$ such that $S^{1} \times N$ admits a symplectic structure $\omega$; then there exists a face $F_{T}$ of the unit ball of the Thurston norm contained in a face $F_{A}$ of the unit ball of the Alexander norm.

This quite peculiar property is satisfied by fibered 3-manifolds, and supports the conjecture that a 3 -manifold $N$ such that $S^{1} \times N$ admits a symplectic structure must in fact be fibered.

Theorems 1.1 and 1.2 hold true also in the case of $b_{1}(N)=1$. It is not surprising that the proof is technically quite longer, due to the chamber structure of the SW invariants in that case. We will omit this case here, referring the interest reader to $[\mathbf{V}]$, where it is treated in detail.

We will then address the problem of determining the constraints for a cohomology class $\alpha$ on a symplectic 4 -manifold to be represented by a symplectic form $\omega$. It is clear that such an $\alpha$ must have positive square, and its pairing with the SW basic classes must satisfy the constraints determined in $[\mathbf{T 2}]$. Li-Liu have conjectured in $[\mathbf{L L}]^{1}$ that these are sufficient conditions. We will prove the following:

Theorem 1.3. There exists a symplectic 4-manifold of the form $S^{1} \times N$ and a cohomology class $\alpha$ of positive square satisfying Taubes' "more constraints" which can not be represented by a symplectic form.

\footnotetext{
${ }^{1}$ Added in proof: The conjecture appears in the preliminary version of $[\mathbf{L L}]$ (see http://www.arxiv.org/abs/math.SG/0012048v1).
} 
Hence, Li-Liu conjecture is false.

\section{Alexander and Thurston norms.}

We start by briefly recalling the definition of Alexander and Thurston norms on the first cohomology group of a closed, oriented 3-manifold $N$. Denote by $F$ the free abelian group $F:=H_{1}(N, \mathbb{Z}) /$ Tor; by definition, $\operatorname{rk}(F)=b_{1}(N)$. The Alexander polynomial of $N$ is an element of the group ring $\mathbb{Z}[F]$, i.e., a finite sum

$$
\Delta_{N}=\sum_{\mathbf{i}} a_{\mathbf{i}} t^{\mathbf{i}}
$$

where $\mathbf{i}=\left(i_{1}, \ldots, i_{b_{1}(N)}\right)$ is a multi-index of cardinality $b_{1}(N), t=\left(t_{1}, \ldots\right.$, $\left.t_{b_{1}(N)}\right)$ with $\left\{t_{i}\right\}$ a basis of $F$ and $a_{\mathbf{i}}$ are integer coefficients. The Alexander polynomial is well-defined up to multiplication by units of $\mathbb{Z}[F]$. For any element $\phi \in H^{1}(N ; \mathbb{Z})$ we define the norm

$$
\|\phi\|_{A}:=\max _{\mathbf{i j}} \phi\left(t^{\mathbf{i}} \cdot t^{-\mathbf{j}}\right),
$$

where the indexes run over all $\mathbf{i}, \mathbf{j}$ such that $a_{\mathbf{i}}, a_{\mathbf{j}}$ are nonzero. It is clear that this definition is unaffected by the indeterminacy in the Alexander polynomial and does not depend on the coefficients.

The Thurston norm, described in [Th2], is defined as follows: For any Riemann surface $\Sigma$ embedded in $N$ denote

$$
\chi_{-}(\Sigma)=\sum_{\Sigma_{i} \mid g\left(\Sigma_{i}\right) \geq 1}\left(-\chi\left(\Sigma_{i}\right)\right),
$$

where $\Sigma$ is the disjoint union of the $\Sigma_{i}$; we then define the norm

$$
\|\varphi\|_{T}=\min \left\{\chi_{-}(\Sigma) \mid \Sigma \hookrightarrow N, \operatorname{PD}[\Sigma]=\phi\right\} .
$$

It is not difficult to verify that both norms are linear on rays and satisfy the triangle inequality. It is possible to continuously extend these norms to cohomology with real coefficients. The unit ball of these norms is then a finite, convex (possibly noncompact) polyhedron. In particular, the unit ball of the Alexander norm is by construction dual (up to a factor 2) to the Newton polyhedron of $\Delta_{N}$.

\section{Basic classes and monopole classes.}

In this section we will discuss the way the Alexander and Thurston norms are related to Seiberg-Witten theory. Essentially the relation between Alexander norm and SW theory will be deduced from Meng-Taubes proof of the equivalence of a SW invariant of a 3-manifold and the Alexander polynomial of the manifold. The relation of Thurston norm and SW theory, instead, has been analyzed in $[\mathbf{K M}]$. 
We start with a brief review of SW theory in dimension three, in order to have a formulation which is the suitable for our purposes. Let $(N, g)$ be a smooth, closed, oriented, riemannian three dimensional manifold. We will assume that $b_{1}(N)>1$. We equip $N$ with the canonical homology orientation induced by a basis of $F$. Once $N$ is endowed with a $\operatorname{spin}^{c}$ structure $P_{N}$, i.e., a $U(1)$-lifting of the $S O(3)$ frame bundle, we can consider the three dimensional SW equations

$$
F_{A}=q(\psi)-i \eta, \quad \not_{A} \psi=0,
$$

where $A$ is a connection on the determinant bundle of the $\operatorname{spin}^{c}$-structure, $q(\cdot)$ is an $\Omega^{2}(N ; i \mathbb{R})$-valued bilinear form on the sections of the spinor bundle associated to $P_{N}, \eta$ is a perturbation term that lives in $\Omega^{2}(N ; \mathbb{R}) \cap$ ker $d$, and $\not_{A}$ is the Dirac operator that acts on spinors. These equations are invariant under the gauge group of those automorphisms of $P_{N}$ which act trivially on the frame bundle. This group acts freely away from reducible couples, that we can remove suitably choosing good perturbations. It is possible to prove (see e.g., $[\mathbf{M T}]$ ), using standard techniques, that choosing a generic nonexact perturbation the moduli space of solutions of Equation (6), modulo gauge equivalence, is a 0-dimensional compact, oriented, smooth manifold; under change of the metric and perturbation (as $\left.b_{1}>1\right)$ different moduli spaces are moreover cobordant. We denote by $\mathcal{M}\left(P_{N}, g, \eta\right)$ the moduli space of solutions of Equations (6), omitting the arguments whenever unnecessary.

We define the SW invariant for $P_{N}$ as the algebraic sum of the oriented points of $\mathcal{M}\left(P_{N}, g, \eta\right)$ for $\eta$ a good perturbation. We have the following definition:

Definition 3.1. Let $c \in H^{2}(N ; \mathbb{Z})$ be an integral cohomology class that arises as first Chern class of a spin ${ }^{c}$-structure $P_{N}$ such that the invariant $\mathrm{SW}\left(P_{N}\right)$ is nonzero. Then $c$ is called a basic class of $N$.

It is quite clear from this definition that the SW equations for a basic class admit a solution for any metric and a generic perturbation. Moreover, as the compactness of the equations implies that non-emptiness is an open condition, also the unperturbed equations have a solution for any metric, i.e., $\mathcal{M}\left(P_{N}, g, 0\right) \neq \emptyset$ (note that this space can be nonsmooth). This makes it natural to introduce the:

Definition 3.2 (Kronheimer-Mrowka). Let $c \in H^{2}(N ; \mathbb{Z})$ an integral cohomology class that arises as Chern class of a $\operatorname{spin}^{c}$-structure $P_{N}$ such that $\mathcal{M}\left(P_{N}, g, 0\right) \neq \emptyset$ for any metric $g$. Then $c$ is called a monopole class.

From the previous observation, the set of monopole classes, that we denote by $\mathcal{C}(N)$, contains all the basic classes.

We now introduce, following ref. $[\mathbf{M T}]$, an element in $\mathbb{Z}[[F]]$, defined from the family of SW invariants of the $\operatorname{spin}^{c}$-structures. 
The set $\mathcal{S}$ of $\operatorname{spin}^{c}$-structures on $N$ is an affine $H^{2}(N ; \mathbb{Z})$. There is a natural way to define a map from $\mathcal{S}$ to $F$, constructed as follows: Fix a reference $\operatorname{spin}^{c}$-structure $Q_{N}$, that we choose to be the product structure. Any other structure $P_{N}$ differs from it by the action of an element of $H^{2}(N, \mathbb{Z})$. Consider now the composed map

$$
H^{2}(N, \mathbb{Z}) \stackrel{\mathrm{PD}}{\longrightarrow} H_{1}(N, \mathbb{Z}) \stackrel{\pi}{\longrightarrow} F .
$$

Using this map we can construct a map $s$ which goes from $\mathcal{S}$ to $F$. The fiber of this map is given by the order of the torsion of $H_{1}(N ; \mathbb{Z})$, that we will denote now on by ord $(N)$. Note that twice this map gives, up to torsion, the Poincaré dual of the Chern classes of $P_{N}$. Consider for any $t^{\mathbf{i}} \in F$ the set $s^{-1}\left(t^{\mathbf{i}}\right) \in \mathcal{S}$. These are the $\operatorname{spin}^{c}$-structures that have the same real Chern class. Define now

$$
\mathrm{SW}\left(t^{\mathbf{i}}\right):=\sum_{s^{-1}\left(t^{\mathbf{i}}\right)} \mathrm{SW}\left(P_{N}\right)
$$

we can now define from this the function

$$
\mathrm{SW}(N)=\sum_{\mathbf{i}} \mathrm{SW}\left(t^{\mathbf{i}}\right) t^{\mathbf{i}} \in \mathbb{Z}[[F]] .
$$

Well-known facts of SW theory are that the number of $\operatorname{spin}^{c}$-structures for which unperturbed SW equations admit solutions is bounded, and that the invariant $\mathrm{SW}\left(t^{\mathrm{i}}\right)$ is symmetric under the natural involution of $F$. These observations, together with the definition of the function SW, yield the fact that $\mathrm{SW}(N)$ is a symmetric element of $\mathbb{Z}[F]$.

The previous definition, in the case where $\operatorname{ord}(N)=1$, is a simple reformulation of SW theory. In the other cases, instead, they define a kind of "average" over all structures which have the same real Chern class. We can introduce a new definition that is quite practical for treating the information on $\operatorname{spin}^{c}$-structures contained in the SW functions of Equation (9). For any element $\gamma \in H^{2}(N, \mathbb{Z})$, we denote by $\gamma^{F}$ its projection to $H^{2}(N, \mathbb{Z}) / \operatorname{Tor}\left(=F^{\mathrm{PD}}\right)$.

Definition 3.3. Let $c \in H^{2}(N, \mathbb{Z}) /$ Tor be a cohomology class such that

$$
\sum_{c_{1}^{F}\left(P_{N}\right)=c} \mathrm{SW}\left(P_{N}\right) \neq 0
$$

Then $c$ is called an a-basic class (where the "a" stands for averaged).

We have the following inclusions:

$$
\begin{aligned}
\mathcal{A}(N) & =(\text { a-basic classes }) \subset(\text { basic classes })^{F} \subset(\text { monopole classes })^{F} \\
& =\mathcal{C}(N)^{F} .
\end{aligned}
$$

We want to relate now a-basic classes with the SW function $\mathrm{SW}(N)$ : Let $c$ be an a-basic class; then the sum appearing in Equation (10) coincides with 
$\mathrm{SW}\left(t^{\mathbf{i}}\right)$ where $t^{\mathbf{i}}$ is defined by the relation $t^{2 \mathbf{i}}=\mathrm{PD}(c)$, and the invariant $\mathrm{SW}\left(t^{\mathbf{i}}\right)$ is nonzero.

\section{Relation between the norms.}

Our aim now is to relate a-basic classes of $N$ with its Alexander polynomial, and then to the Alexander norm. In this section we will give a proof of the following:

Proposition 4.1. Let $N$ be a closed three manifold with $b_{1}(N)>1$; then the Alexander norm of an element $\phi \in H^{1}(N ; \mathbb{Z})$ is given by

$$
\|\phi\|_{A}=\max _{\mathcal{A}(N)}(c \cdot \phi)
$$

where the maximum is taken over all a-basic classes of $N$.

Proof. The basic ingredient for the proof is provided by the theorem of Meng and Taubes which identifies the SW function with the (sign-refined) Reidemeister-Franz torsion introduced by Milnor. This is related, on its own, to the (sign-refined) symmetrized Alexander polynomial, denoted by $\Delta_{N}^{s}$. More precisely, we have:

Lemma 4.2 (Meng-Taubes, Turaev). Let $N$ be a closed three manifold equipped with its canonical homology orientation, with $b_{1}(N)>1$; then we have, in $\mathbb{Z}[F]$,

$$
\mathrm{SW}(N)=\Delta_{N}^{s}
$$

From this relation, considering the definitions in Equations (2) and (9), we have $\mathrm{SW}\left(t^{\mathbf{i}}\right)=a_{\mathbf{i}}$ and, by Definition 3.3, a-basic classes $c_{\mathbf{i}} \in \mathcal{A}(N)$ are twice the Poincaré duals of elements of $F$ with nonvanishing invariant $\mathrm{SW}\left(t^{\mathrm{i}}\right)$, i.e.,

$$
c_{\mathbf{i}} \in H^{2}(N ; \mathbb{Z}) / \text { Tor is a-basic } \Longleftrightarrow a_{\mathbf{i}} \neq 0 \text { where } t^{2 \mathbf{i}}=\operatorname{PD}\left(c_{\mathbf{i}}\right) .
$$

The use of the relations of Equation (13) allows us to write the Alexander norm in terms of a-basic classes: We can write

$$
\|\phi\|_{A}=\max _{\mathbf{i j}} \phi\left(t^{\mathbf{i}} \cdot t^{-\mathbf{j}}\right)=\max _{\mathcal{A}(N)}(c \cdot \phi) .
$$

The latter equality comes as follows: For any fixed couple $\mathbf{i}, \mathbf{j}$ with nonzero coefficient we have

$$
\phi\left(t^{\mathbf{i}} \cdot t^{-\mathbf{j}}\right)=\phi\left(t^{\mathbf{i}}\right)-\phi\left(t^{j}\right) \leq \max _{\mathbf{i j}}\left(\left|\phi\left(t^{2 \mathbf{i}}\right)\right|,\left|\phi\left(t^{2 \mathbf{j}}\right)\right|\right) \leq \max _{\mathbf{k}}\left|\phi\left(t^{2 \mathbf{k}}\right)\right|
$$

where $\mathbf{k}$ ranges among all indexes with nonzero coefficient. Being $\Delta_{N}^{s}$ symmetric, equality in Equation (16) is attained for some choice of index with $\mathbf{j}=-\mathbf{i}= \pm \mathbf{k}$. By Equation (14), we can thus write $\phi\left(t^{2 \mathbf{k}}\right)=\left(c_{\mathbf{k}} \cdot \phi\right)$ (and remove the absolute value). This completes the proof of our statement. 
The content of Proposition 4.1 is the good one for our purpose, because of the results of $[\mathbf{K M}]$ on the relations between monopole classes and Thurston norm: We have now all we need to prove Theorem 1.1.

First, we observe that we can restrict the proof to the case of an irreducible manifold. In fact, the SW polynomial vanishes for the connected sum of manifolds with $b_{1}>0$, and the set of a-basic classes of a manifold is preserved for connected sum with a rational homology sphere (the SW polynomial gets multiplied by the order of the torsion of the rational homology sphere).

For irreducible manifolds we have now the equality expressed in:

Theorem 4.3 (Kronheimer-Mrowka). Let $N$ be a manifold as above: Then the Thurston norm of a class $\phi \in H^{1}(N, \mathbb{Z})$ is given by

$$
\|\phi\|_{T}=\max _{\mathcal{C}(N)}(c \cdot \phi) \text {. }
$$

Putting together the inclusion $\mathcal{A}(N) \subset \mathcal{C}(N)^{F}$, Theorem 4.3 and Proposition 4.1, we deduce the inequality (1) for any closed three manifold (for the sole purpose of proving inequality 1.1, it is sufficient at this point to prove an inequality in Equation (17) for all basic classes, as in $[\mathbf{A}]$ ).

Remark. Equation (15) states that the unit ball of the Alexander norm, up to a factor, is dual to the Newton polyhedron of SW $(N)$. Equation (17), in light of the content of $[\mathbf{K M}]$ (see also [K2]) states that the Thurston unit ball is dual, up to the same factor, to the polyhedron of "SWF $(N)$ ", an element of $\mathbb{Z}[F]$ that can be constructed from the Seiberg-Witten-Floer invariants of $N$ (still lacking a rigorous treatment). This answers a longstanding question of Fried in $[\mathbf{F}]$.

\section{Symplectic $S^{1} \times N$.}

In this section we will use the Alexander and Thurston norm to study the following conjecture:

Conjecture 5.1 (Taubes). Let $N$ be a 3-manifold such that $S^{1} \times N$ admits a symplectic structure $\omega$. Then $N$ admits a fibration over $S^{1}$.

We will assume again that $b_{1}(N)>1$. Under this condition manifolds that fiber over $S^{1}$ are irreducible, and it is known that for any class $\phi \in H^{1}(N, \mathbb{Z})$ representing a fibration we have $\|\phi\|_{A}=\|\phi\|_{T}$ (see e.g., $[\mathbf{M c M}]$ ). Fibered classes are known to satisfy the following condition (see [Th2]): The integral points laying in the cone over a (top dimensional) face of the Thurston unit sphere have the property of being all fibered, or none does. This implies in particular that a fibered face of the unit ball of Thurston norm is contained in a face of the unit ball of the Alexander norm.

We would like to prove that the latter condition holds for any $N$ such that $S^{1} \times N$ is symplectic. We will be able to do so under the further assumption 
that $N$ is irreducible; in view of the results of $[\mathbf{M c C}]$, this is a reasonable assumption. Our proof adapts to the case of $b_{1}>1$ the strategy of [K1].

We observe that, as simplecticity is an open condition, there is no restriction (see $[\mathbf{D}]$ ) in assuming that the symplectic form $\omega$ on $S^{1} \times N$ is the reduction of an integer class of $H^{2}\left(S^{1} \times N, \mathbb{Z}\right)$. There is a cone, in $H^{2}\left(S^{1} \times N, \mathbb{R}\right)$, of cohomology classes that can be represented by symplectic forms, and in this cone the set of classes which are in the image of the cohomology with rational coefficients is dense. We will be interested to have cohomology classes that lie in the image of the cohomology with integral coefficients, and eventually pass to sufficiently high multiples of the symplectic form: We will implicitly assume this whenever necessary.

We want to recall now some general results that we will apply to our case. The first is the Donaldson theorem on the existence of symplectic submanifolds $([\mathbf{D}])$. This theorem assures that there exist a connected symplectic submanifold $H \subset S^{1} \times N$ such that

$$
[H]=\mathrm{PD}[\omega]=\left[S^{1}\right] \times \gamma+\tau \in H_{2}\left(S^{1} \times N, \mathbb{Z}\right)
$$

where $\gamma \in H_{1}(N, \mathbb{Z}), \tau \in H_{2}(N, \mathbb{Z})$ and $\gamma \cdot \tau>0$ (for sake of notation we will denote all products, both on $N$ and on $S^{1} \times N$, with a dot, the distinction being clear from the context). Denote $\phi=\operatorname{PD}(\tau) \in H^{1}(N, \mathbb{Z})$; as a consequence of the previous discussion, the $\phi$ 's associated to symplectic forms as in the relation of Equation (18) define a cone in $H^{1}(N, \mathbb{Z})$.

The second result is that the $\operatorname{spin}^{c}$-structures on $S^{1} \times N$ with nontrivial SW invariants must be pull backs of $\operatorname{spin}^{c}$-structures on $N$ (to prove this you can use, e.g., the adjunction inequality); moreover there is an identification between the moduli spaces for a $\operatorname{spin}^{c}$-structure $P_{N}$ on $N$ and the moduli space for the pull-back structure on $S^{1} \times N$ (that we will usually denote with the same symbol), once a suitable correspondence of the perturbation terms is set (see $[\mathbf{O T}]$ ). This allows the identification, up to a sign determined by the choice of homology orientations, of the SW invariants associated to these moduli spaces.

The third point concerns $\operatorname{spin}^{c}$-structures on a symplectic four manifold $(M, \omega)$ with canonical bundle $K$. There exist, in that case, a canonical spin $^{c}$-structure that decomposes as $\mathbb{C} \oplus K^{-1}$ (and has first Chern class equal to $-K)$. Any other $\operatorname{spin}^{c}$-structure can be written as $E \oplus\left(K^{-1} \otimes E\right)$ for an $E \in H^{2}(M, \mathbb{Z})$. There are some constraints on spin ${ }^{c}$-structures with nonvanishing invariants that arises from Taubes' work (see [T1], [T2]). In the case of $b_{+}(M)>1$ the canonical spin ${ }^{c}$-structure has SW invariant \pm 1 and for any other structure $E_{i} \oplus\left(K^{-1} \otimes E_{i}\right)$ with nonzero invariants we have $K \cdot \omega \geq E_{i} \cdot \omega \geq 0$. Equality implies, respectively, $E_{i}=\mathbb{C}$ or $E_{i}=K$. 
This inequality translates, for the basic classes $\kappa_{i}:=\operatorname{det}\left(E_{i} \oplus\left(K^{-1} \otimes E_{i}\right)\right)$, in the relation

$$
K \cdot \omega \geq\left|\kappa_{i} \cdot \omega\right|,
$$

with equality only for the case $\kappa_{i}= \pm K$. Let's apply these results to $S^{1} \times N$. First, the canonical class and all other basic classes are pull backs: There exists a preferred line bundle $K \in H^{2}(N, \mathbb{Z}$ ) (for sake of simplicity, we use the same notation on $N)$ and a preferred $\operatorname{spin}^{c}$-structure on $N$ of the form $\mathbb{C} \oplus K^{-1}$ with SW invariant \pm 1 such that any other $\operatorname{spin}^{c}$-structure on $N$ appears as $E_{i} \oplus\left(K^{-1} \otimes E_{i}\right)$ for $E_{i} \in H^{2}(N, \mathbb{Z})$. The structures with nonzero invariants must satisfy $K \cdot \phi \geq E_{i} \cdot \phi \geq 0$, the equalities implying respectively $E_{i}=\mathbb{C}$ or $E_{i}=K$. This translates to a constraint, for the basic classes of $N$, which has the form

$$
K \cdot \phi \geq\left|\kappa_{i} \cdot \phi\right|,
$$

with equality only for the case $\kappa_{i}= \pm K$.

Using this it is straightforward to prove the following:

Proposition 5.2. Let $\left(S^{1} \times N, \omega\right)$ be a symplectic manifold with $b_{1}(N)>1$, and denote by $\phi \in H^{1}(N, \mathbb{Z})$ the Künneth component of $[\omega]$; then $\|\phi\|_{A}=$ $K \cdot \phi$. Moreover, $\phi$ lies in the cone over a top dimensional face of the unit ball of the Alexander norm, dual to the vertex $K$ of the Newton polyhedron of $\Delta_{N}$.

Proof. The maximum of $\kappa_{i} \cdot \phi$ for $\kappa_{i}$ basic is attained for and only for $K$. We want to use this property to evaluate the Alexander norm, in the form expressed in Proposition 4.1. To do this we need only to prove that $K$ (or, more precisely, its image $K^{F}$ in $H^{2}(N, \mathbb{Z}) /$ Tor) is an a-basic class. But no other basic class $\kappa_{i}$ can coincide up to torsion with $K$ without violating Equation (20), so that the sum of Equation (10), namely $\sum_{c_{1}^{F}\left(P_{N}\right)=K^{F}} \mathrm{SW}\left(P_{N}\right)$, contains only one nonzero term, that term being equal to 1 . This means that $K^{F}$ is an a-basic class. We can conclude, following Proposition 4.1, that $\|\phi\|_{A}=K \cdot \phi$. The rest of the proposition is an obvious consequence of what was previously stated.

We will use Proposition 5.2 to write the genus of the symplectic submanifold $H$ of Equation (18), in conjunction with the adjunction inequalities for manifolds of type $S^{1} \times N$ that are contained in [K1]. These apply to irreducible manifolds $N$ which do not have a basis of $H_{2}(N, \mathbb{Z})$ composed of tori. Leaving aside this totally degenerate case, for which the equality of Alexander and Thurston norm is trivial, we have the following:

Proposition 5.3. Let $\left(S^{1} \times N, \omega\right)$ be a symplectic manifold with $N$ irreducible, $b_{1}(N)>1$, and denote by $\phi \in H^{1}(N, \mathbb{Z})$ the Künneth component of $[\omega]$ : Then $\|\phi\|_{A}=\|\phi\|_{T}$. 
Proof. The adjunction inequality for embedded submanifolds of $S^{1} \times N$ of [K1] can be written in the form

$$
\chi_{-}(H) \geq H \cdot H+\|\phi\|_{T}=2 \gamma \cdot \tau+\|\phi\|_{T} .
$$

As $H$ is symplectic, the adjunction formula for symplectic submanifolds gives

$$
\chi_{-}(H)=H \cdot H+K \cdot H=2 \gamma \cdot \tau+\|\phi\|_{A} .
$$

These formulae are compatible with the content of Equation (1.1) if and only if $\|\phi\|_{A}=\|\phi\|_{T}$.

We can somehow strengthen this result. By Equation (1.1) the unit ball of the Thurston norm is contained in the unit ball of the Alexander norm; it is clear that extending the Alexander norm to real coefficients, and using the denseness of $H^{1}(N, \mathbb{Q})$, the equality stated in Proposition 5.3 continues to hold in an open cone of $H^{1}(N, \mathbb{R})$ determined by the cone of classes of $H^{2}\left(S^{1} \times N, \mathbb{R}\right)$ admitting a symplectic representative (the norm is a continuous function). Therefore a (top dimensional) face $F_{T}$ of the unit ball of the Thurston norm (containing $\phi /\|\phi\|_{T}$ ) intersects a face $F_{A}$ of the unit ball of the Alexander norm (the face dual to $K$ ); but this implies that the entire $F_{T}$ is contained in $F_{A}$.

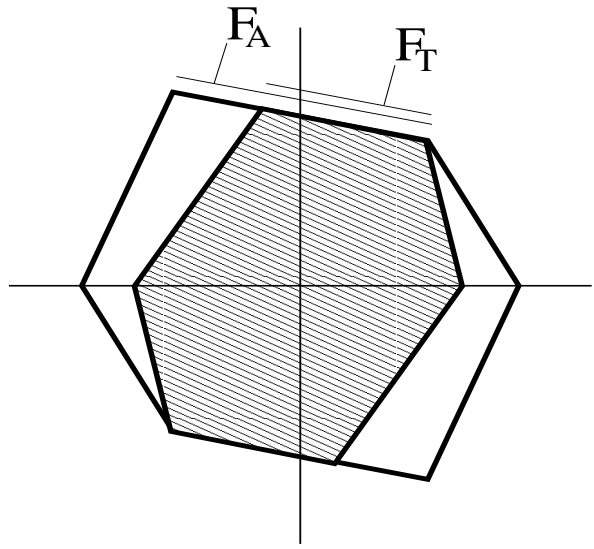

Figure 1. Alexander unit ball and Thurston unit ball for a 3-manifold such that $S^{1} \times N$ is symplectic.

Figure 1 shows a possible case of the relation between the norms for $N$ as described.

This observation completes the proof of Theorem 1.2. 


\section{Representability of a cohomology class by a symplectic form.}

A classical problem of symplectic topology is to determine necessary and sufficient conditions for a 2-cohomology class $\alpha$ on an even-dimensional closed, smooth, oriented manifold $M$ admitting an almost complex structure with canonical class $K$, to be represented by a symplectic form. This problem merges with the general problem of the existence of any symplectic structure on $M$. Necessary conditions arise from the very definition of symplectic form; in particular, if $M$ has dimension 4, we need $\alpha \in \mathcal{P}$, where

$$
\mathcal{P}=\left\{\beta \in H^{2}(M, \mathbb{R}) \mid \beta \cdot \beta>0\right\} .
$$

An early conjecture, in [Th1], speculated that every almost complex manifold with nonempty $\mathcal{P}$ admitted a symplectic structure. Since then, other constraints have been identified. In particular, more refined conditions arise from Taubes' constraints on SW basic classes: As mentioned, we must have $\mathrm{SW}(K)= \pm 1$, and the class $\alpha$ must satisfy the conditions of Equation (19). We denote by $\mathcal{T}$ the cone composed of elements of $\mathcal{P}$ satisfying these constraints, i.e.,

$$
\mathcal{T}:=\left\{\alpha \in \mathcal{P}|K \cdot \alpha \geq| \kappa_{i} \cdot \alpha \mid\right\},
$$

with strict inequality when $\kappa_{i} \neq \pm K$.

It is well-known that satisfying Taubes' constraints is not a sufficient condition for $\alpha$ to be represented by a symplectic form. In fact, as discussed in $[\mathbf{K M T}]$, if we consider the manifold $X \# \Sigma$ where $X$ is symplectic and $\Sigma$ is an homology 4-sphere admitting a nontrivial cover, and the cohomology class $\alpha_{\omega}$ on $X \# \Sigma$ induced by a symplectic form $\omega$ on $X$ under the natural isomorphism $H^{2}(X, \mathbb{R})=H^{2}(X \# \Sigma, \mathbb{R})$, we have identity of the SeibergWitten polynomials $\mathrm{SW}_{X}=\mathrm{SW}_{X \# \Sigma}$ and $\alpha_{\omega}$ lies in $\mathcal{T}_{X \# \Sigma}$, but cannot be represented by a symplectic form for the simple reason that $X \# \Sigma$ itself does not admit symplectic structures (it has a cover with trivial SW polynomial). There is another class of potential, more refined, examples of couples $(M, \alpha)$ satisfying these constraints with $\alpha$ not representable by a symplectic form. These are knot surgery manifolds homotopic to a $K 3$ surface (see [FS] for the definition) obtained from a knot $K$ : It is commonly conjectured that whenever $K$ is not a fibered knot, $M$ can not be symplectic, but it is easy to find nonfibered knots such that Taubes' constraints are satisfied for a class $\alpha$.

In both the previous cases, the absence of a symplectic form representing $\alpha$ has to be attributed, in some sense, to the manifold $M$ (which does not admit tout court symplectic structures) and not to the cohomology class itself. We can ask about the situation for manifolds known to be symplectic. In particular, it has been conjectured (see $[\mathbf{L L}]$, Section 4) that if we assume that $X$ is a symplectic manifold, the cone $\mathcal{T}$ coincides with the "symplectic 
cone"

$$
\mathcal{W}:=\{\alpha \in \mathcal{P} \mid \alpha \text { is represented by a symplectic form }\} .
$$

The conjecture gives a possible answer to the following problem, outlined in the beginning of this section, namely:

Question. Let $M$ be a symplectic manifold. Determine the cone, in $H^{2}(M, \mathbb{R})$, represented by symplectic forms.

Some partial answer to this question are known. For example, Geiges proved in $[\mathbf{G}]$ that for $T^{2}$-bundles over $T^{2}$, all classes in the positive cone are represented by symplectic forms (we remark that all these classes satisfy Taubes' constraints, as the canonical class is trivial); it is interesting also to compare with the result of Gromov for the case of open manifolds, where any form in the positive cone lies in $\mathcal{W}$.

Concerning this Question, we have the following result:

Theorem 6.1. There exist symplectic manifolds, of the form $S^{1} \times N^{3}$, on which there are cohomology classes of positive square satisfying Taubes' constrains but which can not be represented by symplectic forms, i.e., the strict inclusion $\mathcal{W} \subset \mathcal{T}$ holds true. In particular, the conjecture of $[\mathbf{L L}]$ is false.

Proof. The proof is based on the following assumption, that will be proved in the next section (Theorem 7.5): There exists a family of fibered 3-manifolds, whose generic component is denoted by $N$, with $H_{1}(N, \mathbb{Z})=\mathbb{Z}^{2}$, such that the fibered face $F_{T}$ of the Thurston unit ball is strictly contained in the face $F_{A}$ of the Alexander unit ball. Assuming this, we proceed as follows. Denote by $\mathcal{V}$ the (nonempty) cone, in $H^{1}(N, \mathbb{R})$, over $F_{A} \backslash \bar{F}_{T}$. Choose a $\phi \in \mathcal{V}$. We claim that we can define an a class $\psi \in H^{2}(N, \mathbb{R})$ such that the cohomology class $\alpha \in H^{2}\left(S^{1} \times N, \mathbb{R}\right)$ with Künneth decomposition

$$
\alpha=\phi \wedge[d t]+\psi
$$

has positive square and satisfies Taubes' constraints, i.e., $\alpha \in \mathcal{T}$. This is achieved in the following way. We have $\alpha \cdot \alpha=2 \phi \cdot \psi$ : Identify $H^{2}(N, \mathbb{R})=$ $\operatorname{Hom}\left(H^{1}(N, \mathbb{R}), \mathbb{R}\right) ;$ to get a positive square, we can choose $\psi$ to be any element of the cone $\operatorname{Hom}\left(\phi, \mathbb{R}_{+}\right)$. We observed in Section 5 that the basic classes on $S^{1} \times N$ are pull-back of basic classes on $N$; the choice of $\psi$ is therefore irrelevant for the constraints of Equation (24) and $\alpha$ belongs to $\mathcal{T}$ if and only if $\phi$ satisfies the condition (on $\left.H^{*}(N, \mathbb{R})\right) K \cdot \phi \geq\left|\kappa_{i} \cdot \phi\right|$ with equality only for the case $\kappa_{i}= \pm K$. But this condition is equivalent to the condition that $\phi$ lies in the cone over $F_{A}$, as $F_{A}$ is, by definition, the face dual to $K$, i.e., the elements lying in the cone over $F_{A}$ have maximal pairing, among all basic classes, with and only with $K$.

To complete the proof, we need to show now that $\alpha$ can not be represented by a symplectic form. By Proposition 5.3 (and the following comments, if we want to work with cohomology with real coefficients), if $\alpha$ admits 
a symplectic representative, then its Künneth component $\phi$ should have the same Alexander and Thurston norm, something we excluded choosing $\phi \in \mathcal{V}$.

Note that proceeding as above we can without difficulty choose the class $\alpha$ to lie in the image of cohomology with integer coefficients.

\section{Remarks.}

1. The symplectic manifold discussed in Theorem 6.1 is not simply connected, but we believe that there exist simply connected examples. In particular, we expect that the link surgery manifolds obtained using a link with fibered face strictly contained in a face of the Alexander norm (as the one we will discuss in the next section), are possible examples. The difficulty in proving such result arises from the difficulty of proving the analogue of Proposition 5.3 (see Section 7 of [K2] for a discussion of this interesting problem).

2. The failure of Li-Liu conjecture, as expressed in the examples of Theorem 6.1 , is due to the mismatch between the convex hull of basic classes and the convex hull of monopole classes, as the latter determines the extension of the fibered cone of $H^{1}(N, \mathbb{R})$. It is coincevable to improve the conjecture, at least for symplectic manifolds of the form $S^{1} \times N$, by reformulating the definition of the cone $\mathcal{T}$ as

$$
\mathcal{T}:=\left\{\alpha \in \mathcal{P}|K \cdot \alpha \geq| \kappa_{i} \cdot \alpha \mid \text { for any monopole class } \kappa_{i}\right\},
$$

with strict inequality when $\kappa_{i} \neq \pm K$. In that case, as follows from the results of [Th2], the conjecture would hold true assuming the validity of a strict version of Conjecture 5.1, which takes the form:

Conjecture 6.2. Let $N$ be a 3 -manifold such that $S^{1} \times N$ admits a symplectic structure $\omega$; then the Künneth component of $[\omega]$ in $H^{1}(N, \mathbb{R})$ can be represented by a nondegenerate 1 -form (i.e., it lies in a fibered cone).

\section{Construction of the three manifolds.}

In this section we will justify the assumption made in the proof of Theorem 6.1, namely the existence a family of closed, fibered 3-manifolds with the property that $F_{T}$ is strictly contained in $F_{A}$. Our construction will be based on the existence of a noteworthy 2-component link, exhibited by Dunfield in $[\mathbf{D u}]$, which has the same property. We will need the following result:

Proposition 7.1 (Dunfield). There exists a 2-component oriented link $D=$ $D_{1} \cup D_{2} \subset S^{3}$ with Alexander polynomial

$$
\Delta_{D}\left(t_{1}, t_{2}\right)=\left(t_{1}-1\right)\left(t_{2}-1\right)
$$


(written in terms of the homology classes of the meridians to the two components) which has a fibered face $F_{T}$ strictly contained in a face $F_{A}$, dual to the vertex $t_{1} t_{2}^{-1}$ of the dual polyhedron.

(In Dunfield's paper, the Alexander polynomial and the norms are discussed in terms of an homology basis different from ours, but it is easy to rewrite them in terms of the standard homology basis for the link exterior, as above.)

We don't know the exact shape of the Thurston unit ball, but for our purpose it is enough to know the result contained in the previous Proposition. Denote $N_{D}=S^{3} \backslash \nu\left(D_{1} \cup D_{2}\right)$ :

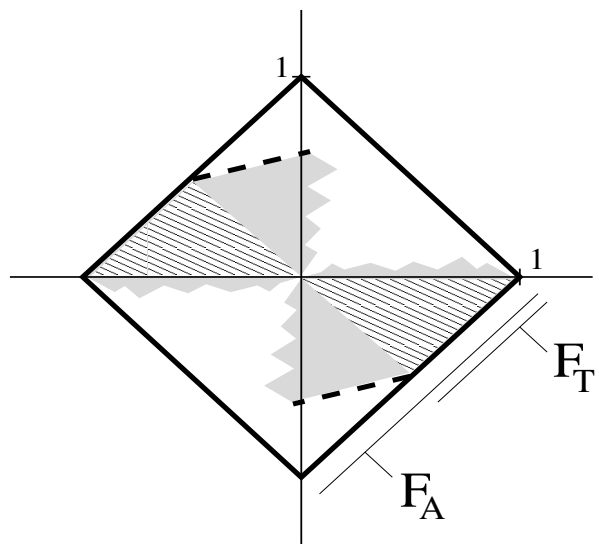

Figure 2. Alexander unit ball and Thurston unit ball for $N_{D}$ — the dotted regions are qualitative.

Figure 2 represents, in the space $H^{1}\left(N_{D}, \mathbb{R}\right)$ with basis vectors the dual basis $\tau_{i}$, the unit ball of the Alexander norm, and a part of the unit ball of the Thurston norm.

Let now $K_{1}, K_{2}$ be a couple of fibered knots of genus $g\left(K_{i}\right)>0$ and let $\Delta_{K_{i}}(t)$ be their Alexander polynomials. Next, define the closed manifold

$$
N\left(K_{1}, K_{2}\right)=N_{D} \cup\left(\coprod_{i=1}^{2} S^{3} \backslash \nu K_{i}\right),
$$

where on the boundary tori the gluing map is defined to be the orientation reversing diffeomorphism which identifies the basis $\left(\mu\left(D_{i}\right), \lambda\left(D_{i}\right)\right)$ with $\left(\mu\left(K_{i}\right),-\lambda\left(K_{i}\right)\right)$. To interpret this, notice that each knot exterior is an homology solid torus, so that this operation appears as an homology Dehn filling for $N_{D}$, with surgery coefficient 0 . The reason of the choice of this surgery curve appears evident from the fact (for a proof, see [EN], Section 3) that the minimal genus Seifert surface in $N_{D}$ representing an homology class Poincaré dual to a class $\left(m_{1}, m_{2}\right) \in H^{1}\left(N_{D}, \mathbb{Z}\right)$ intersects the boundary 
torus $T_{i}$ in $m_{i}$ copies of the longitude of each link component (note that $\left.l k\left(D_{1}, D_{2}\right)=\Delta_{D}(1,1)=0\right)$. Each minimal genus Seifert surface of $N_{D}$ has therefore a natural capping in $N\left(K_{1}, K_{2}\right)$, given by the union of $m_{i}$ copies of the fiber of $S^{3} \backslash \nu K_{i}$. In particular, if $\left(m_{1}, m_{2}\right)$ is a fibered class, this fibration extends to a fibration of $N\left(K_{1}, K_{2}\right)$ through the fibrations

$$
S^{3} \backslash \nu K_{i} \longrightarrow S^{1} \stackrel{(\cdot)^{m_{i}}}{\longrightarrow} S^{1}
$$

of the knots' exteriors. This proves, in particular, that $N\left(K_{1}, K_{2}\right)$ is irreducible. As the linking number of $D$ is zero, $H_{1}\left(N\left(K_{1}, K_{2}\right), \mathbb{Z}\right)=\mathbb{Z}^{2}$, canonically identified with $H_{1}\left(N_{D}, \mathbb{Z}\right)$.

It is natural to guess that, for any class $\left(m_{1}, m_{2}\right) \in H^{1}\left(N_{D}, \mathbb{Z}\right)$, the surface constructed above is the minimal genus representative for the cohomology class Poincaré dual to $\left(m_{1}, m_{2}\right) \in H^{1}\left(N\left(K_{1}, K_{2}\right), \mathbb{Z}\right)$. This is confirmed by the following:

Lemma 7.2 (Eisenbud-Neumann, Prop. 3.5). If $M$ is a compact irreducible 3 -manifold and $\mathbf{m} \in H^{1}(M)$, then the Thurston norm $\|\mathbf{m}\|_{T}$ is the sum of the norms of the restrictions of $\mathbf{m}$ to the Jaco-Shalen-Johannson components of $M$.

As a consequence of this Lemma, denoting with the symbol $\|\cdot\|_{\hat{T}}$ the norm on the closed manifold, we have the following:

Corollary 7.3. The Thurston norm $\left\|\left(m_{1}, m_{2}\right)\right\|_{\hat{T}}$ of an element $\left(m_{1}, m_{2}\right) \in$ $H^{1}\left(N\left(K_{1}, K_{2}\right), \mathbb{Z}\right)$ is given by

$$
\left\|\left(m_{1}, m_{2}\right)\right\|_{\hat{T}}=\left\|\left(m_{1}, m_{2}\right)\right\|_{T}+\left|m_{1}\right|\left(2 g\left(K_{1}\right)-1\right)+\left|m_{2}\right|\left(2 g\left(K_{2}\right)-1\right)
$$

where $\left\|\left(m_{1}, m_{2}\right)\right\|_{T}$ is the Thurston norm of the corresponding element of $N_{D}$.

Proof. This follows from Lemma 7.2, together with the observation that the class $\left(m_{1}, m_{2}\right)$ on the closed manifold restricts to the element with same coordinates in $N_{D}$ and to the classes $m_{i} \in H^{1}\left(S^{3} \backslash \nu K_{i}, \mathbb{Z}\right)$, which have Thurston norm $\left\|m_{i}\right\|_{T}=\left|m_{i}\right|\left(2 g\left(K_{i}\right)-1\right)$, by definition of genus of a knot and linearity on rays.

We want to study now the Alexander norm of the manifold $N\left(K_{1}, K_{2}\right)$; in order to do this we need a gluing formula for the Alexander polynomial (or the SW invariant) along tori. We have the following:

Lemma 7.4 (Gluing formula). Let $N\left(K_{1}, K_{2}\right)=N_{D} \cup\left(\coprod_{i=1}^{2} S^{3} \backslash \nu K_{i}\right)$ be defined as above: Then the Alexander polynomials of the manifolds are related by the formula

$$
\Delta_{N\left(K_{1}, K_{2}\right)}\left(t_{1}, t_{2}\right)=\Delta_{N_{D}}\left(t_{1}, t_{2}\right) \frac{\Delta_{K_{1}}\left(t_{1}\right)}{t_{1}-1} \frac{\Delta_{K_{2}}\left(t_{2}\right)}{t_{2}-1}=\Delta_{K_{1}}\left(t_{1}\right) \Delta_{K_{2}}\left(t_{2}\right) .
$$


Therefore, the Alexander norm $\|\cdot\|_{\hat{A}}$ on $N\left(K_{1}, K_{2}\right)$ is given by

$$
\begin{aligned}
\left\|\left(m_{1}, m_{2}\right)\right\|_{\hat{A}} & =\left|m_{1}\right| 2 g\left(K_{1}\right)+\left|m_{2}\right| 2 g\left(K_{2}\right) \\
& =\left\|\left(m_{1}, m_{2}\right)\right\|_{A}+\left|m_{1}\right|\left(2 g\left(K_{1}\right)-1\right)+\left|m_{2}\right|\left(2 g\left(K_{2}\right)-1\right) .
\end{aligned}
$$

Proof. It is known (see $[\mathbf{M T}],[\mathbf{T u}]$ ) that the Milnor torsion is multiplicative by gluing along tori, with suitable identification of the variables; this torsion coincides with the Alexander polynomial for manifolds having $b_{1}>1$ and it is equal to the Alexander polynomial $\Delta_{K}(t)$ divided by $(t-1)$ for the case of a knot. Remembering Proposition 7.1, Equation (32) above follows. The relation on the Alexander norm is then an easy corollary of this formula, as the degree of the Alexander polynomial of a fibered knot equals twice its genus.

This Lemma says, in particular, that the unit ball of the Alexander norm for $N_{D}$ and $N\left(K_{1}, K_{2}\right)$ are conformally equivalent (see Figure 3 ). We are ready to prove:

Theorem 7.5. There exist a family of fibered closed 3-manifolds $N$ with $H_{1}(N, \mathbb{Z})=\mathbb{Z}^{2}$ such that a fibered face of the Thurston unit ball is strictly contained in the corresponding face of the Alexander unit ball.

Proof. Our family is given by $N\left(K_{1}, K_{2}\right)$ for any choice of the fibered knots $K_{i}$. We observed before that each fibration of $N_{D}$ in $F_{T}$ extends to a fibration of $N\left(K_{1}, K_{2}\right)$, defining a fibered face $F_{\hat{T}}$ of the Thurston unit ball for $N\left(K_{1}, K_{2}\right)$. This face will be contained in $F_{\hat{A}}$, one of the four faces of the Alexander unit ball (having the same cone as $F_{A}$ ). This face is dual to the vertex $t_{1}^{2 g\left(K_{1}\right)} t_{2}^{-2 g\left(K_{2}\right)}$ (square of a vertex of the Newton polyhedron of the symmetrized Alexander polynomial). If a class $\left(m_{1}, m_{2}\right) \in H^{1}\left(N_{D}, \mathbb{Z}\right)$ lies in the cone over $F_{A} \backslash \bar{F}_{T}$ (in particular $\left\|m_{1}, m_{2}\right\|_{A}<\left\|m_{1}, m_{2}\right\|_{T}$ ), then the corresponding class on the closed manifold has Alexander norm strictly smaller than the Thurston norm, from Equations (31) and (33), i.e., $F_{\hat{T}}$ is strictly contained in $F_{\hat{A}}$. From this the statement follows.

Figure 3 describes the Thurston and Alexander norm for a particular choice of $g\left(K_{i}\right)$.

We want to outline a second proof of the same statement, based on the fact that a class on a closed three manifold is fibered if and only if all the restrictions to each JSJ component are fibered (see [EN], Theorem 4.2): If the Thurston norm on the closed manifold coincided with the Alexander norm on a larger cone than the one on $N_{D}$, then there would be fibered classes on $N\left(K_{1}, K_{2}\right)$ which restrict, on $N_{D}$, to nonfibered ones (as mentioned above, all integral points laying on a face of the unit ball of the Thurston norm containing at least one fibration are fibered).

We finish this section pointing out that, although the link $D$ above is the only example worked out in detail, fibered links with the properties of 


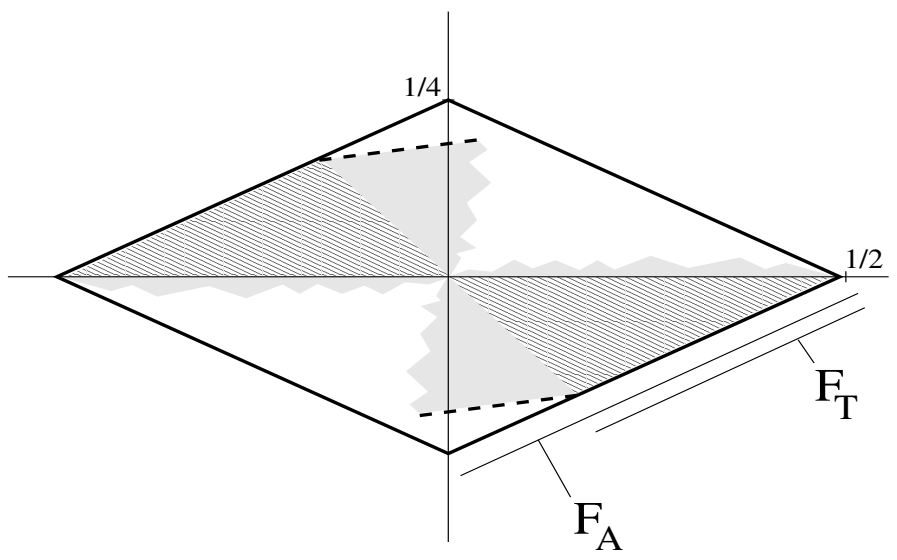

Figure 3. Alexander unit ball and Thurston unit ball for $N\left(K_{1}, K_{2}\right)$ with $g\left(K_{1}\right)=2, g\left(K_{2}\right)=4$ - the dotted regions are qualitative.

Proposition 7.1 are likely to be "frequent" (compare the discussion in [Du]). From these examples, other closed 3-manifolds can be constructed.

\section{References}

[A] D. Auckly, The Thurston norm and three-dimensional Seiberg-Witten theory, Osaka J. Math., 33 (1996), 737-750, MR 97j:57045, Zbl 0881.57034.

[D] S. Donaldson, Symplectic submanifolds and almost-complex geometry, J. Diff. Geom., 44 (1996), 666-705, MR 98h:53045, Zbl 0883.53032.

[Du] N. Dunfield, Alexander and Thurston norm of fibered 3-manifolds, Pacific J. Math., 200(1) (2001), 43-58, CMP 1863406.

[EN] D. Eisenbud and W. Neumann, Three Dimensional Link Theory and Invariants of Plane Curve Singularities, Annals of Mathematics Studies, 110, 1985, MR 87g:57007, Zbl 0628.57002.

[FS] R. Fintushel and R. Stern, Knots, links, and 4-manifolds, Invent. Math., 134 (1998), 363-400, MR 99j:57033, Zbl 0914.57015.

[F] D. Fried, Fibrations over $S^{1}$ with pseudo-Anosov monodromy, Travaux de Thurston sur les Surfaces, Asterisque, 66-67 (1979), 251-265, Zbl 0446.57023.

[G] H. Geiges, Symplectic structures on $T^{2}$-bundles over $T^{2}$, Duke Math. J., 67 (1992), 539-555, MR 93i:57036, Zbl 0763.53037.

[KMT] D. Kotschick, J. Morgan and C. Taubes, Four manifolds without symplectic structures but with nontrivial Seiberg-Witten invariants, Math. Res. Lett., 2 (1995), 119-124, MR 96i:57024, Zbl 0853.57020.

[K1] P. Kronheimer, Minimal genus in $S^{1} \times M^{3}$, Invent. Math., 135 (1999), 45-61, MR 2000c:57071, Zbl 0917.57017. 
[K2] Embedded Surfaces and Gauge Theory in Three and Four Dimensions, Surveys in Differential Geometry, Vol. III (Cambridge, MA, 1996), International Press, 1998, 243-298, MR 2000a:57086, Zbl 0965.57030.

[KM] P. Kronheimer and T. Mrowka, Scalar curvature and Thurston norm, Math. Res. Lett., 4 (1997), 931-937, MR 98m:57039, Zbl 0892.57011.

[LL] T. Li and A. Liu, Uniqueness of symplectic canonical class, surface cone and symplectic cone of 4-manifolds with $b^{+}=1$, J. Differential Geom., 58(2) (2001), 331-370, CMP 1913946.

[McC] J. McCarthy, On the asphericity of a symplectic $M^{3} \times S^{1}$, Proc. Amer. Math. Soc., 129 (2001), 257-264, MR 2001c:57024, Zbl 0972.53045.

$[\mathrm{McM}]$ C. McMullen, The Alexander polynomial of a three manifold and the Thurston norm on cohomology, Ann. Sci. École Norm. Sup. (4), 35(2) (2002), 153-171, CMP 1914929.

[MT] G. Meng and C.H. Taubes, $S W=$ Milnor torsion, Math. Res. Lett., 3 (1996), 661-674, MR 98j:57049, Zbl 0870.57018.

[OT] C. Okonek and A. Teleman, 3-dimensional Seiberg-Witten invariants and nonKählerian geometry, Math. Ann., 312 (1998), 261-288, MR 2000d:57050, Zbl 0909.57010.

[T1] C. Taubes, The Seiberg-Witten invariants and symplectic forms, Math. Res. Lett., 1 (1994), 809-822, MR 95j:57039, Zbl 0853.57019.

[T2] - More constraints on symplectic forms from Seiberg-Witten invariants, Math. Res. Lett., 2 (1995), 9-13, MR 96a:57075, Zbl 0854.57019.

[Th1] W.P. Thurston, Some simple examples of symplectic manifolds, Proc. Amer. Math. Soc., 55 (1976), 467-468, MR 53 \#6578, Zbl 0324.53031.

[Th2] - A norm for the homology of 3-manifolds, Mem. Amer. Math. Soc., 59(339) (1986), 99-130, MR 88h:57014, Zbl 0585.57006.

[Tu] V. Turaev, Reidemeister torsion in knot theory, Russian Math. Surveys, 41 (1986), 119-182, MR 87i:57009, Zbl 0602.57005.

[V] S. Vidussi, The Alexander norm is smaller than the Thurston norm: A SeibergWitten proof. Prepublication École Polytechnique, 6, 1999.

Received July 12, 2001 and revised January 26, 2002.

Department of Mathematics

UNIVERSITY OF CALIFORNIA

IRVINE, CA 92697

Department of Mathematics

Kansas State University

Manhattan, Kansas 66506

E-mail address: vidussi@math.ksu.edu 